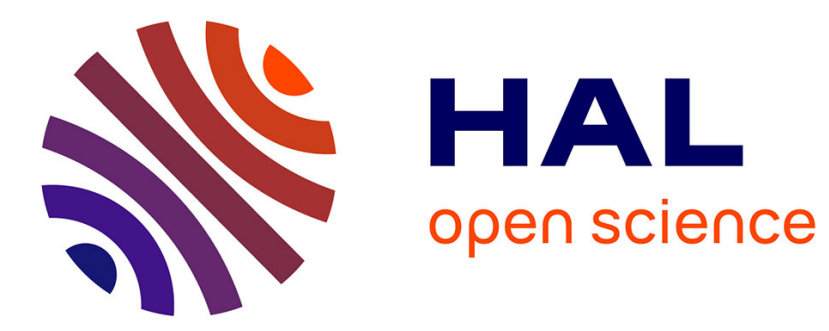

\title{
Simulating multiple stars in preparation for Gaia
}

Frédéric Arenou

\section{To cite this version:}

Frédéric Arenou. Simulating multiple stars in preparation for Gaia. International Workshop on Double and Multiple Stars: Dynamics, Physics, and Instrumentation, Dec 2009, Saint-Jacques-deCompostelle, Spain. pp.107-121, 10.1063/1.3597593 . hal-02054993

\section{HAL Id: hal-02054993 https://hal.science/hal-02054993}

Submitted on 2 Mar 2019

HAL is a multi-disciplinary open access archive for the deposit and dissemination of scientific research documents, whether they are published or not. The documents may come from teaching and research institutions in France or abroad, or from public or private research centers.
L'archive ouverte pluridisciplinaire HAL, est destinée au dépôt et à la diffusion de documents scientifiques de niveau recherche, publiés ou non, émanant des établissements d'enseignement et de recherche français ou étrangers, des laboratoires publics ou privés. 


\title{
Simulating multiple stars in preparation for Gaia
}

\author{
F. Arenou \\ GÉPI, Observatoire de Paris, CNRS, Université Paris Diderot ; \\ 5 Place Jules Janssen, 92190 Meudon, France
}

\begin{abstract}
The scientific preparation of the Gaia mission encompasses both the data reduction algorithms and the generation of simulated data which have to be as realistic as possible. In this respect, binaries and multiple stars are a mandatory component in the simulation of the Milky Way as they impact on the performance tests of the on-ground data processing. The ingredients for the simulation of multiple stars are described and the predictions are compared to observed data or outcomes from stellar multiplicity studies.
\end{abstract}

Keywords: Binaries, Gaia

PACS: 97.80.-d, 95.75.-z

\section{INTRODUCTION}

The Gaia mission, planned to be launched in 2012 by ESA, will provide the most accurate and precise astrometric (but not only astrometric) Catalogue to come for about one billion objects.

The Data Processing and Analysis Consortium [DPAC, 1], about 400 scientists and engineers from the European member states in charge of the future data reduction of the data from the satellite, is organised since 2006 in eight currently active Coordination Units, one of these being in charge of the data simulations. The reason for the presence of a dedicated simulation unit is the complexity both of the observed objects and of the astrometric, photometric, spectroscopic on-board instruments and their interrelations.

Accordingly, the Simulation Unit contains a "Universe Model" and an "Instrument Model", and the former intends to generate the parameters (position, motion, photometry, spectra) of all (or most of the) objects which would be seen by the Gaia satellite at any time of the mission. Correct simulations are mandatory: it can be easily understood that the density of stars has an impact on the instantaneous processing capabilities onboard, that the integrated number of stars during several hours influences the on-board storage needs or the telemetry rate, or that the kind of objects impacts on the requirements for the on-ground data processing.

The objects to be simulated are the solar system objects (planets and asteroids), the extragalactic objects (resolved or unresolved galaxies, quasars, etc), but of course the vast majority of the objects brighter than $G=20^{\mathrm{m}}$ in the Gaia broad band are stars. Most of them are not single. Optical doubles may occur, as typically there is one chance on 700 that more than one star can fit in a $6 \times 12$ pixels window on the average on the sky, up to one chance in 6 in the worst case (the $\approx 3$ millions $G<20$ stars per square degree in the Baade window), and even possibly due to the superposition of the two fields of view of the satellite in the common focal plane. However, most of non-single

\footnotetext{
International Workshop on Double and Multiple Stars: Dynamics, Physics, and Instrumentation

AIP Conf. Proc. 1346, 107-121 (2011); doi: 10.1063/1.3597593

(C) 2011 American Institute of Physics 978-0-7354-0902-6/\$30.00
} 
stars will be actual binaries or multiple systems, about $48 \%$ of systems not being single, although this does not mean at all that they may be detected by any of the instruments (for a description of the algorithms for the detection and data reduction of the multiple systems, see [2], now superseding [3]).

Thanks to the Besançon Galaxy Model (BGM) [4], an accurate simulation of the Galaxy is used as the foundation of the Gaia simulator, but concerning single stars only. Gaia will however notice or suffer from the effect of physical double and multiple stars in astrometry, photometry and spectroscopy, and it is desirable not only to simulate their orbital motion but also to complement the BGM as exactly as possible with the various population properties of what is currently known about multiple stars.

For this reason, simulations of binaries have been introduced in the Gaia simulator for long [5]. In a first step, solar-type stars-like binaries were introduced only, using the classical results from Duquennoy and Mayor [6]. Yet, the simulations were not satisfactory enough for several reasons: the single star fraction was severely underestimated towards low mass stars, multiple stars were not handled, and the resulting luminosity function was not consistent with the mass distribution. Accordingly, the generation of the secondaries (or more) components has been improved to achieve a more realistic simulation of the Galaxy.

\section{The recipe}

The BGM generates single stars only and when a new star is drawn, it can then be changed with some probability into a system, the BGM allowing to draw a companion of a given mass on the HR diagram, coeval of the primary. The single stars and primaries are obviously chosen such that they follow the luminosity function of primaries in the solar neighborhood, not the one of unresolved binaries.

Using the primary mass, the mass of the companion is then obtained through a given statistical relation $q=M_{2} / M_{1}=f\left(M_{1}\right)$, a "primary-constrained pairing" process [8]. Beyond the preparation steps of this recipe, helped by [9], what matters really is the nature and quantities of the ingredients, all this being described below.

\section{MULTIPLICITY FRACTION}

The main observational effect of binaries come from their fraction relative to the total population. While a single number is often quoted ("more than half of stars are in binary systems"), many studies have been done since Duquennoy and Mayor [6]. Thanks to

the recently available statistics for what concerns multiplicity, all binaries cannot be considered to be just like the solar-type ones, and this has led Lada [10] to provocatively argue that most stars of the Galaxy are single, due to the low binary frequency of the most frequent star type. 


\section{Binary frequency}

In what follows, binary stars, or $2+$, often designate the systems having at least two components, and more generally, following Tokovinin [11], $a_{k}$ denotes the fraction of systems containing at least $k$ components.

The binary fraction is discussed below as a function of the primary star. While, observationally, the primary of a system is conventionally the brightest, the other convention is used for operational (orbital) purposes, i.e. the primary will always be the one with the largest mass, and consequently the generated mass ratio is constrained to be $0 \leq q \leq 1$, and components of a system may have to be swapped to respect this principle.

\section{Main sequence stars}

Concerning Main Sequence (MS) solar type stars, the results from [6] have slowly been improved by studies using different range of periods. The statistics for the F7-K from Eggenberger et al. [12] is $55.6 \%$ for $\log \left(P_{\text {days }}\right)<6.31$ and we adopt this value as the total fraction over the whole period/separation range.

Towards the Very Low Mass stars (VLM) side, the binary fraction is decreasing, to about $28 \%$ for the $\mathrm{M}$ stars [13], 15\% for the low mass $\mathrm{M}$ [14], 12.5\% from a complete sample in distance of the ultracool L-dwarf population [15], and 9\% for T brown dwarfs [16], probably underestimated (but not too much, see Figs. 3 a and 4) as this T dwarfs statistics concerns separations $a \gtrsim 1$ AU and mass ratio $q \gtrsim 0.4$.

While the current scientific interest is often directed towards solar type stars down to VLM, finding unbiased results about the binarity properties of high-mass stars is still uneasy. For example, a $91 \pm 12 \%$ binary fraction for $7 M_{\odot}$ stars may be found in Sterzik and Durisen [17]; however, this value is actually extrapolated, assuming a constant distribution in $\log a$ (commonly known as Öpik's law), from the $0.20 \pm 0.04$ companion star fraction (mean number of companion per star) per decade of separation found by Shatsky and Tokovinin [18] for B-type stars over a 0.3"-6.4" separation range in Sco OB2.

Still in Sco OB2, Kouwenhoven et al. [19] find a detected binary fraction $\approx 80 \%$ for B0-B3 stars, $\approx 50 \%$ for B4-B9 stars, $\approx 40 \%$ for A-type stars. They demonstrate however that the decrease may be due to observational biases not taken into account. Later, correcting the results from incompleteness, Kouwenhoven et al. [20] estimate that a $85-100 \%$ binary fraction for stars of spectral type B and $80-85 \%$ for those of spectral type A would better fit their data. Using the lower limit, we assume $85 \%$ for earliest B stars $\left(20 M_{\odot}\right)$ and $80 \%$ for earliest A stars $\left(3 M_{\odot}\right)$. A similar result (a binary fraction from 0.8 to 1) is obtained in Cygnus OB2 by Kobulnicky and Fryer [21].

The reason why we use the lower limit is that Mason et al. [22] comparing the binarity in cluster/association, field or runaway O-type stars, suggests that almost all O-stars in clusters and associations have companions but that the binarity is much lower for field stars and still lower for the runaways. In the solar-type mass range, Halbwachs et al. [23] comparing field and cluster stars show that the binary fraction for the latter is slightly larger though not significantly. Beside, the completeness is probably better in clusters. 
All in all, we consider not useful to adopt various proportions whether a star is in a cluster or not and the adopted fraction is actually a (not completely satisfactory, and still not well known however) compromise.

We thus adopt the binary probability function

$$
f\left(M_{1}\right)=0.8388 \tanh \left(0.688 M_{1}+0.079\right)
$$

which fits rather well the binary fraction on the whole primary mass range (Fig. 1). The shape of this function has not been chosen randomly but as roughly compatible with the several classes of dynamical decay models from Sterzik and Durisen [17] or random pairing from Thies and Kroupa [24]. However, the increase with primary mass over the short period solar type range which is assumed here deviates from the conclusion of Halbwachs et al. [23] (finding no obvious proportion difference from F7 to late K) though perhaps not significantly.

Binary fraction vs primary mass

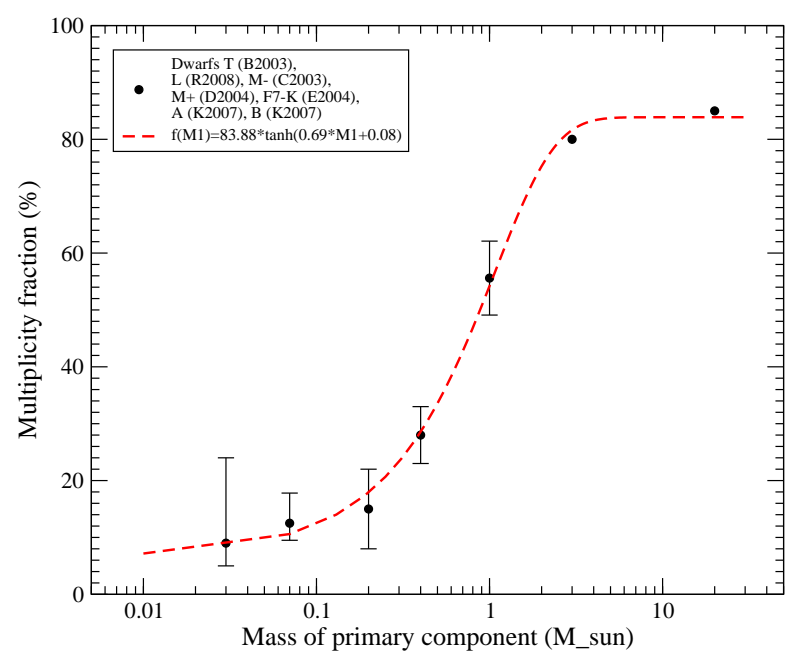

FIGURE 1. Binary fraction: the observed estimated fractions for different primary masses found in the literature (dots) and the fit proposed. From left to right, the points from the literature were obtained from Burgasser et al. [16], Reid et al. [15], Close et al. [14], Delfosse et al. [13], Eggenberger et al. [12], and Kouwenhoven et al. [20] for A and B type stars.

A large discrepancy on the high mass side still remains between the large adopted fraction here and the one indicated in Eggleton and Tokovinin [25] (ET2008). There are several reasons to believe that this illustrates selection effects mentioned in the latter, which uses complete samples, but in magnitude, not distance, and, unfortunately, most observation methods act against the binarity detection in early-type stars. Typically, the broad lines in $\mathrm{O}$ and $\mathrm{B}$ stars prevents a good detection of the radial velocity changes due to binarity; on the astrometric binary side, this is not better, as the systems are generally too distant for an efficient astrometric orbital detection; and, on the visual side, couples with intermediate orbital periods and somewhat large magnitude difference may also be too close to be detected. Still, as discussed above, most of other studies indicate that the 
bias-corrected multiplicity fraction of early-type stars should be more on the $100 \%$ than on the $50 \%$ side [e.g. 26, 22, 18, 19, 20, 21].

\section{White dwarfs}

Main sequence stars, although in larger numbers, are not the only ones which produce specific observational effects with Gaia. Obtaining a good representation of the white dwarfs (WD) in the binary model has been triggered by the need of simulating light curves that will be obtained with Gaia due to cataclysmic variables.

As white dwarfs in simulated binary systems occur either because a) the primary is a WD and a secondary has subsequently been chosen, or because b) the secondary happens to be a WD with some probability (cf. p. 6), obtaining a correct number of (realistic!) systems at the end is complicated.

In the case (a), the probability of having a secondary has to be fixed, although the binary WD fraction cannot be a single number as it also depends on period and companion type. We actually adopted the binary rate that the WD progenitor had while being on the main sequence, as described Eq. 11, the progenitor mass being given as a function of the WD mass [obtained in 34].

Concerning case $b$ ), the probability for the companion of being a WD has been based on the frequency of white dwarfs in the BGM. In practice, it has been used from the ratio of the WD fraction over the star fraction (A. Robin, private communication) which in turns depends on the age populations used in the model. Care should be taken however that the progenitor of the WD should have evolved faster than the primary (which was formerly the secondary), based on an estimation of its mass.

To judge whether WD systems were correctly handled there are fortunately some tests based on the fraction of systems with one WD obtained with ROSAT [30] or Barstow et al. [31], on the fraction of double degenerates (WD+WD) among systems with WD or on Sirius-like binaries (A0-K7+WD) from Holberg et al. [32], and the density of possible cataclysmic variables (CV) per parsec cube [33] where a CV is defined here for operational purposes as a WD in a system with period $<0.6$ day.

\section{Giants}

The knowledge of the multiplicity fraction for giants has recently been improved, thanks to better statistical studies based on distance estimate from Hipparcos and CORAVEL radial velocity survey. Following the results initially given by Famaey et al. [27], Mermilliod et al. [28] studied the K giants and Frankowski et al. [29] the M giants. For the latter, an upper bound for the binarity fraction is 0.111 ((confirmed ) +0.027 (suspected), apparently corresponding to a 0.43 fraction of the former, which gives an upper bound $(32 \%)$ for the binarity of the $\mathrm{K}$ giant, still quite uncertain as it varies from either $13.7 \%$ [27] or $22 \%$ [28] in the field, or 30.8\% in open clusters.

However, the fractions quoted above have been obtained for Spectroscopic Binaries (SB), i.e. short periods only and the completeness in period not really being indicated. 
Extrapolating these results for the whole period range based on some model (described p. 7) would add a considerable uncertainty on the adopted numbers.

Consequently, we proceeded as for the WD, adopting the Eq. 1 fraction, noting that the period distribution further decreases the fraction by rejecting unphysical systems.

\section{Higher multiplicity fraction}

Concerning now the simulation of higher levels of multiplicity, the adopted fractions have been mostly based on the extensive work by Tokovinin, and in particular from Eggleton and Tokovinin [25] (ET2008). First, it can be noted that the $a_{k}$ curves for the various $k$ are rather similar (Table 6 in ET2008) as a function of spectral type or $M_{1}$. Consequently, the fractions of $3+$ may be obtained using the $a_{2}\left(M_{1}\right)$ curve, i.e. generating the binaries and then adding a tertiary component.

The frequency of ternary systems among binaries is however strongly related to period, as can be seen in Tokovinin et al. [35], p. 129: among solar type SBs with periods less than 30 days, $a_{3} / a_{2}=86 \%$ of the systems with period below 5 days harbour a tertiary component versus $49 \%$ for those with a larger $(5<P<30$ days) inner period. To get the $3+$ fraction for the rest of inner periods, it has been assumed that the total fraction of triples $a_{3}$ for solar type stars is $8.4 \%$, [6], and using the assumed distribution of periods (p. 7), $a_{3} / a_{2}=11 \%$ was assumed for $P>30$ days.

For the 4+ systems, the relation between the multiplicity fractions mentioned above is shown Fig. 2. Although this ratio $a_{k} / a_{2}$ is obtained in ET2008 for a large $V<6$ magnitude-limited sample, we adopted an $\exp ^{-1.087(N-2)}$ fit to their probably more complete $V<4$ sub-sample to generate the $4+$, samples from the $3+$ one, and the same fraction to get the $5+$ from the $4+$ number.



FIGURE 2. Observed probability to get systems with $N+$ component given that there are at least 2 components, from the ET2008 catalogue of multiplicity limited to magnitude 4 or 6 . The fit on the former has been adopted to estimate the $4+$ and $5+$ fractions. 


\section{MASSES AND ORBITAL PARAMETERS}

\section{Mass-ratio}

Once it is known that the star is actually a system, this star becomes the primary, and the secondary is generated through the choice of a mass-ratio, which, as indicated above, is constrained to be smaller than 1 . The secondary mass being given, it can be chosen with some probability as a MS, giant or WD, depending on the age of the primary.

The adopted mass ratio has been estimated by rejection simulation using a probability density function linear by segment, and this, depending on the stellar type of the primary, and on the binary period, as illustrated Fig. 3. These simulations have been based on the works from (by increasing mass) [36] for VLM, from [13, Fig. 4] for the M stars with period smaller or larger than 50 days, by [23, Fig. 7] for the F7-K stars with period smaller or larger than 50 days, and by [18, Fig. 9] for OB stars. The peak at $q=1$ which historically has always been a matter of debate (as it may be produced e.g. by a photometric selection bias of twins) seems to be present at small periods, and it may even decrease with mass [37]. Concerning this point, but also more generally, the simulated models should absolutely not be taken at face value as there is a considerable statistical uncertainty in the published results. Still, the simulated mass-ratios are better than a uniform distribution which would be adopted otherwise!


FIGURE 3. Simulated mass-ratio $q=M_{2} / M_{1}$ for (top to bottom): (a) very-low mass stars, (b) M stars with period $P<50$ days or (c) $P>50$ days, (d) solar type stars with $P<50$ days, (e) solar type with $P>50$ days, and (f) OB stars. See text for bibliographical references.

The mass ratio for the systems with a WD primary has also to be fixed. As it is unknown, the mass ratio from the progenitor has been used instead, as described above. If this gives birth to a companion more massive than what became a WD, the system is rejected as it is already accounted for by the WD secondaries, except when the mass of the secondary was such that it had become a WD too. 
While the secondary is chosen based on the mass-ratio and with the same age, it is however checked that the pairing is realistic (e.g. a pre main sequence star will be bound to another one).

Concerning multiple (3+) systems, the $q_{3}$ mass-ratio may observationally be larger than 1 , on the average $5 \%$ [38], but $18 \%$ for systems with a spectroscopic inner couple [35], which then creates A-BC systems.

\section{Semi-major axis}

Beside their masses, the effect of binaries on astrometry, spectroscopy or photometry is mostly related to their period, or alternatively through the Kepler third law, to their separations.

For the distribution of semi-major axis $a$ (A.U.), the classical Gaussian( $\log a ; 1.5,1.5)$ distribution for solar type stars [6] looks still realistic and has been used here. However, $M$ stars not only have a smaller binary frequency, but the separations are also smaller on the average. Separations are even smaller for ultracool binaries, Bouy et al. [39] showing that most binaries have separations smaller to 20 A.U. We thus assume a Gaussian $(\log a ; 0.5,0.5)$ from Close et al. [14]. In between, it is estimated visually from Fig. 2 of Sterzik and Durisen [17] that a Gaussian $(\log a ; 1,1)$ can be applied to the $\mathrm{M}$ stars (Fig. 4). On the other side of the H-R diagram, and lacking other statistics, we still assume the solar type separation properties for more massive stars.

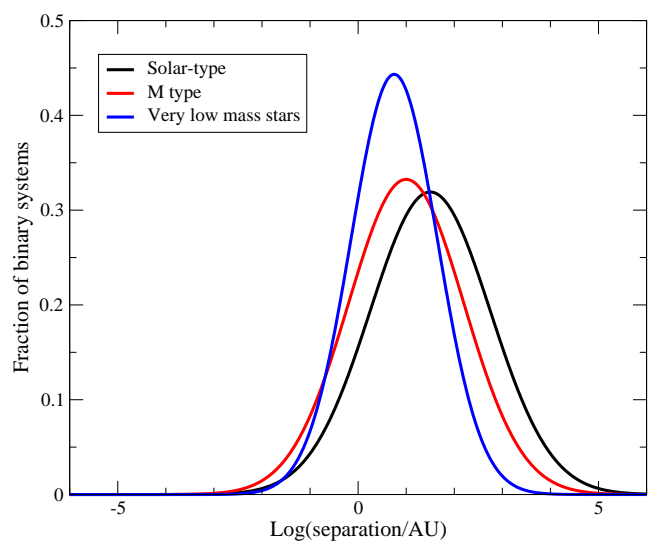

FIGURE 4. Distribution of the separations $(\log (\mathrm{UA}))$ as they are simulated. The broader curve on the right is the classical Duquesnoy \& Mayor distribution, very low mass stars on the left [39] and M stars interpolated in between.

The Gaussian shape implies that very large separations are very rarely generated, but the small separations have also to be taken care of (see close binary systems, p. 9). Once the separation is randomly generated that way, it is combined with the masses to obtain the orbital period. 
Most multiple systems are hierarchical, due to dynamical stability constraints, with a clear limit on the period ratios, $5<P_{\text {out }} / P_{\text {in }}<10^{4}$, [see [40, 38], and the $P_{\text {out }}$ is thus generated from a Gaussian $(\log P ; 6,1.5)$ until it is within the limit above, and the semimajor axis follows from that.

All multiple systems are however not hierarchical: although a lot of known trapezium systems ${ }^{1}$ actually appear like that only in projection or are optical systems [41], about $0.1 \%$ of the IDS Catalogue could still be Trapezia, all with primary type earlier than B3, but much less than originally predicted by Allen et al. [42]. Using Table 5 of this paper and the above result, it has been assumed that less than $10 \%$ of systems more massive than $7 \mathrm{M}_{\odot}$ and with large separations could be trapezium systems.

\section{Eccentricity}

The orbital eccentricity is known from long to depend on period, at least because tidal effects lead to a circularisation of the orbit at small separations, cf. e.g. Abt [43] and Abt [44]. From Abt [44], the average eccentricity can be written:

$$
E[e]=a(b-\exp (-c \log P))
$$

with the $a, b, c$ coefficients given by Abt for B0-B9.5, A0-A5, A6-F0, F0-F9, G0-G9, K0-M5 and giants. Then, the eccentricity is simulated uniformly within the interval $[0,2 E[e]]$, possibly redrawing when larger than 1 .

\section{Remaining orbital parameters}

The other orbital parameters are then drawn randomly. Random does not mean uniform, though. While the periastron date $T$ is indeed chosen uniformly between 0 and the period $P$, the argument of the periastron $\omega_{2}$ uniformly in $[0,2 \pi]$, the position angle of the node $\Omega$ uniformly in $[0,2 \pi]$, the inclination has to be random in $\cos i$, not in $i$.

\section{CLOSE BINARY SYSTEMS}

When a random separation is generated, a cutoff is obviously needed for small separations, as a function of the star types. More generally, a Roche model is needed to avoid generating physically unrealistic systems, e.g. an eccentric orbit with an overcontact system, or a fill factor too small to provide a consistent Roche model, etc.

A Gaia DPAC development unit is in charge of the Gaia Eclipsing Binaries (EB) data reduction [see 2]. This represents a very large human involvement to obtain a fully automated process of a complicated task [45] within the DPAC development

1 defined as $3+$ systems where at least 3 separations $\rho_{i}$ are of the same order of magnitude, for example $1 / 3<\rho_{i} / \rho_{j}<3$ 
organisation. The code, named GESSS, is inspired by, and tested against, the WilsonDevinney and Nightfall codes, and, in order to solve an EB system, the code has a simulation first step which takes care of the realism of the generated system.

As it was out of question to implement again this (many man-years) program, we made use of this simulation step for the management of eclipses, and we use GESSS not only for eclipsing systems, but also to check the likelihood of any generated small separation system. When unphysical, the generation of this multiple system is rejected.

\section{VALIDATION TESTS}

The simulations described above have been implemented in Java as are all the algorithms in the Gaia DPAC environment. Although this document does not describe how the simulations are implemented in practice, this section underlines that, beyond the basic unitary tests which are intended to check the correctness of all individual methods, integration tests have also been implemented for validation purposes. These tests are thus used as non-regression tests when any part of the model is changed, even possibly outside of the part implementing the simulation of multiple stars alone.

Although not mandatory, the usefulness of these tests can be understood by a) the complexity of output data generated thanks to a collaborative software development model by several independent developers, b) the statistical nature of the simulated output, c) the nature of the observed data being only projections into the observational domain, thus suffering some selection biases.

Table 1 shows the various tests which have been implemented. Sample of stars in all directions are randomly generated, the possible selection functions of the available data published are then applied, and the statistics on the simulated data are compared to the observed statistics, then accepted or rejected (within $3 \sigma$ ). As the selection functions may depend on the spectral class of the star, on its mass, on the period (or separation), or on the mass-ratio (or magnitude difference), most of the features of the simulation have then been meaningfully tested.

The errors bars on the simulation data originates from the sample size, which has to be large enough to get useful statistical error bars, but also limited by the computational resources. To this statistical error should be added the uncertainty for the comparison values, also indicated Table 1 .

A (rather unexpected) indication that the simulations are quite realistic does not come directly from the statistics about main sequence stars (as the observed binary fractions were directly based on various recent studies), but rather from white dwarfs. As indicated above, WD in systems come either as primary or as secondary, and in the latter case their existence depends on the adopted mass-ratio and on the period distribution (with possible rejection). However, the statistics on the simulated data look consistent with the observed statistics, simply adopting the properties of the progenitor while on the main sequence, that is taking into account a normal evolution otherwise not accounted for, the only marginal ad-hoc part being the mass level for the secondaries to evolve also towards the WD step.

Beside, the statistics about giants, although marginally consistent to the upper level of observed properties [29] by chance only, probably indicate the presence of selection 
TABLE 1. Statistics computed on the generated samples, in order to check whether the simulation results (column 4) can recover the observational statistics (column 2) found in the bibliographical references indicated column 3. Quoted (binomial) error bars represent the random part only, the systematic ones, while obviously present, being mostly unknown. The indicated ratios show the original number of stars in the reference studies.

\begin{tabular}{|c|c|c|c|}
\hline Statistics computed on the simulated data & $\begin{array}{l}\text { Reference } \\
\text { value }\end{array}$ & $\begin{array}{c}\text { Biblio. } \\
\text { ref. }\end{array}$ & $\begin{array}{l}\text { Value from } \\
\text { simulation }\end{array}$ \\
\hline Fracti & cf. & idem & idem \\
\hline Fracti & $171 / 249 \pm 0.03$ & [49] & $71.6 \pm 0.5 \%$ \\
\hline Fraction of binary systems within $10 \mathrm{pc}$ & $58 / 249 \pm 0.03$ & $\overrightarrow{49]}$ & $\pm 0.4 \%$ \\
\hline & 14 & {$[\overline{49}]$} & $\pm 0.2 \%$ \\
\hline & & 4 & $\pm 0.1 \%$ \\
\hline Visual $n$ & $1.39 \pm 0.02 \%$ & [48] & $1.49 \pm 0.15 \%$ \\
\hline F7- & $13.5_{-1.6}^{+1.8} \%$ & [23] & $12.9 \pm 2 \%$ \\
\hline F7-1 & $=0.01$ & 1 & $\pm 1.4 \%$ \\
\hline Total F7-K multiple frequency, $\log (P)<6$. & $55.6 \pm 2 \%$ & {$[12$} & $60.6 \pm 0.2 \%$ \\
\hline Short period binary frequency of K giants & $\approx 32.1 \pm 2 \%$ & [29] & $39 \pm 1 \%$ \\
\hline Short period binary fr & $35 / 254 \pm 0.02$ & [29] & $27_{-8}^{+10} \%$ \\
\hline te dwarf being binaries & $\approx 40 / 175 \pm 0.03$ & [30] & $28.9 \pm 0.9 \%$ \\
\hline Fraction of WD+WD among WD & $7 / 122_{-0.02}^{+0.03}$ & [32] & $5.4 \pm 0.6 \%$ \\
\hline Fraction of Sirius-like among WD & $8 / 122_{-0.02}^{+0.03}$ & {$[32]$} & $5.3 \pm 0.6 \%$ \\
\hline Density of possible CVs per $1000 \mathrm{pc}^{3}$ & $0.011_{-0.007}^{+0.023}$ & [33] & $0.005 \pm 0.009$ \\
\hline
\end{tabular}

biases, as the statistics for both MS and WD are consistent with the observations.

\section{CONCLUSION}

The implemented multiplicity model generates the binary proportion as a function of the type and mass of the primary, and an initially single star given as input can give birth to a system. This system has the given star as primary, and a secondary which mass ratio is chosen at random, depending in a not-so-simple manner, as it has been taken for the available statistics, from the spectral type of the primary and the binary period. As indicated above, the mass and age of secondary allow it to obtain physical parameters computed using the Hess diagram distribution in the Besançon model, so with some probability this secondary can also be a white dwarf. Depending on the primary mass, the separation between components is chosen, and the period follows from Kepler third law, unrealistic small separations being avoided by the use of the GESSS code. Drawing the random eccentricity depends on the primary type and period of the system, the other orbital parameters being chosen at random. Finally, ternary systems or more are also present, in accordance with latest fractions known from available observations. In this respect, the model of the Galaxy implemented for Gaia should now have achieved a large degree of realism. Some improvements are still to be done, e.g. to ensure that the variability due to close binaries is compatible with the observations. Indeed, the generation of a large sample of simulated stars compared to various published data represents a meaningful validation of the simulation recipe described above. 
To illustrate the predictions of the simulations, a few graphs are shown below, noting that the graph appearances obviously depend both on the multiple star model described above, and on the BGM properties. First, the temperature and luminosity classes of the components is shown Fig. 5, and the period-eccentricity diagram for all the generated pairs compared to giants is shown Fig. 6, noting that these are the underlying distributions, not those which will be detected.
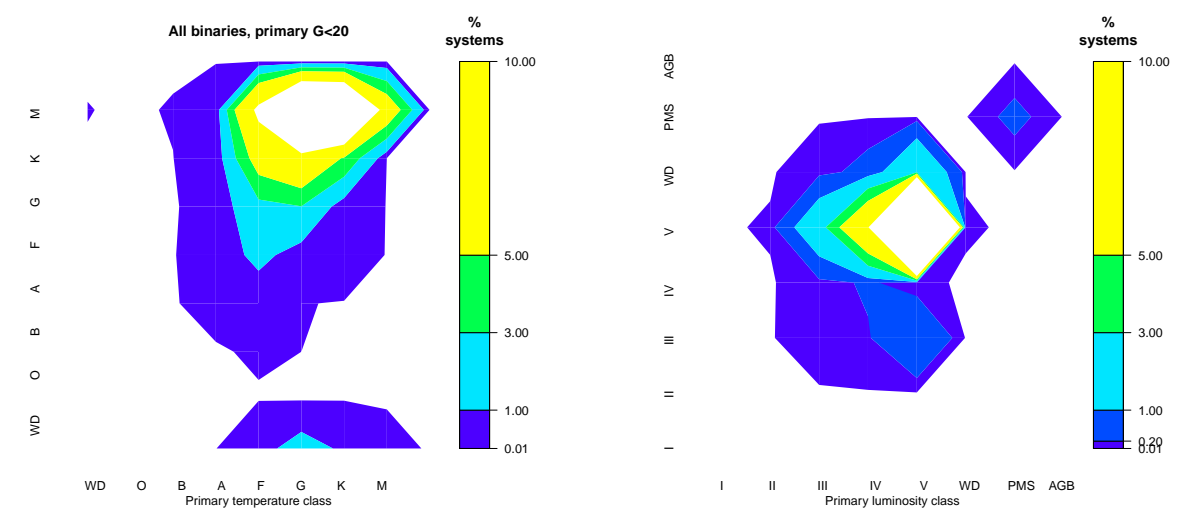

FIGURE 5. Proportion of pairs for the temperature classes (left) and luminosity classes (right): secondary versus primary component for all systems brighter than $G=20$ (binarity detected or not).
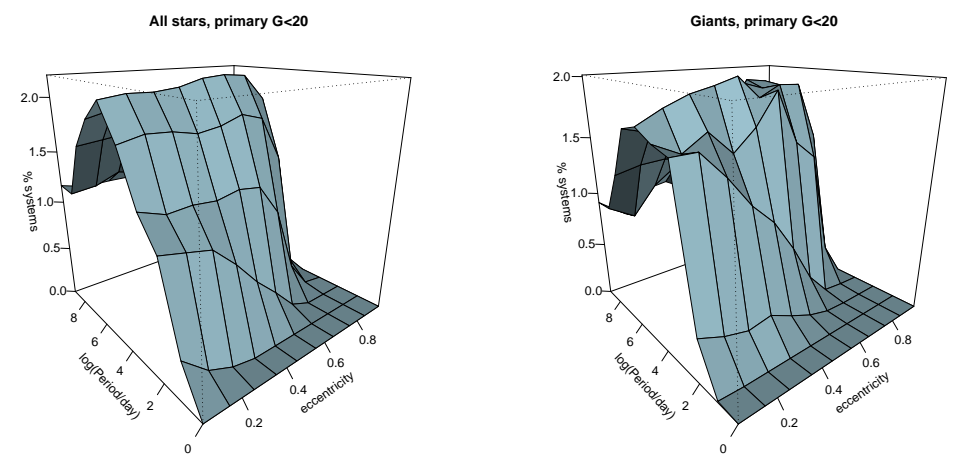

FIGURE 6. The period-eccentricity diagram in a $G<20$ magnitude-limited sample, noting that most pairs may go undetected by the data reduction algorithms, all stars (left), giants only (right, with statistical fluctuations).

Using an update of a formula from Söderhjelm [50], we assume some effective separation capability above which components can be detected $\left(\rho>\rho_{\min }\right.$ depending on magnitude difference and random error based on magnitude plus systematic errors). The mass-ratio distribution shown Fig. 7 indicates how the $q=1$ peak may appear in the detected systems, while it is less present in a volume-limited sample.

Still about visually detected binaries (stressing that millions of other kind of binaries will also be detected through their astrometric, spectroscopic or "photometric" motion), it should be remembered that optical doubles will add to actual visual binaries in high density regions. When a $G<20$ companion is "visually" detected, it is then of interest to estimate the probability of the system to be an optical double or an visual 

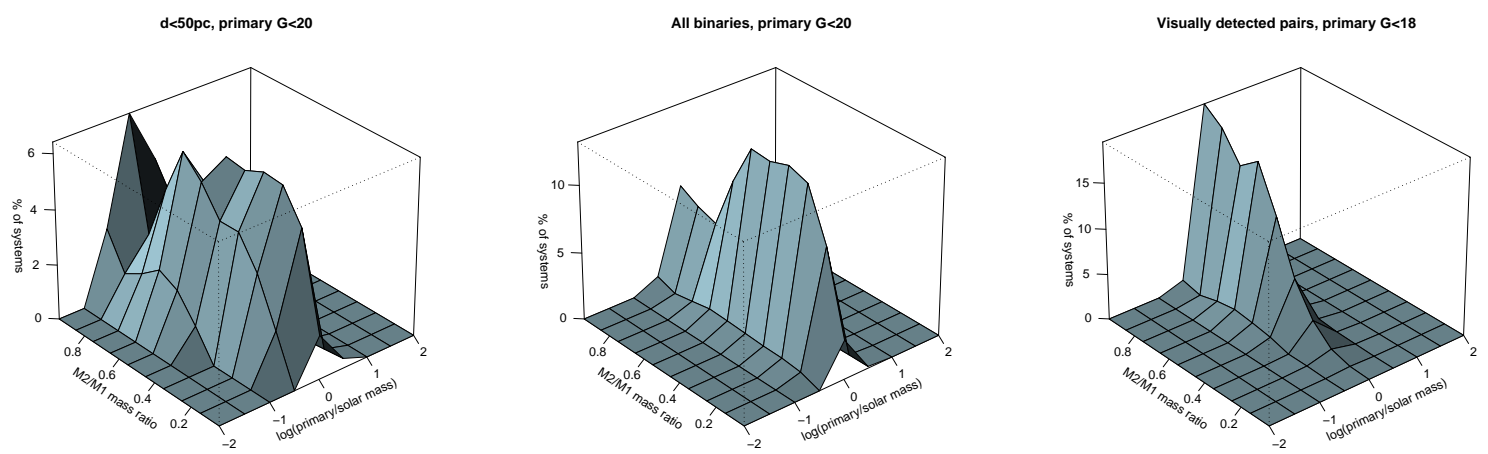

FIGURE 7. Mass ratio versus dex of primary mass for a distance limited sample $(<50$ pc) or a $G<20$ magnitude limited sample assuming that all components could be detected by astrometry, photometry or spectroscopy (far from true!), and a $G<18$ sample where the two components could be "visually" resolved.

binary or multiple system. We assume the detection capability indicated above, and consider as visual double a companion detected within the window of the primary $\left(\rho_{\min }<\rho<1.06\right.$ "). The ratio of visual detections is shown Fig. 8 . Typically, whatever the magnitude, visual binaries will be more numerous outside of the galactic plane, but overwhelmed at the fainter end by optical doubles in the galactic plane (average density $\approx 150000 G<20$ stars per square degree) and towards the galactic center.

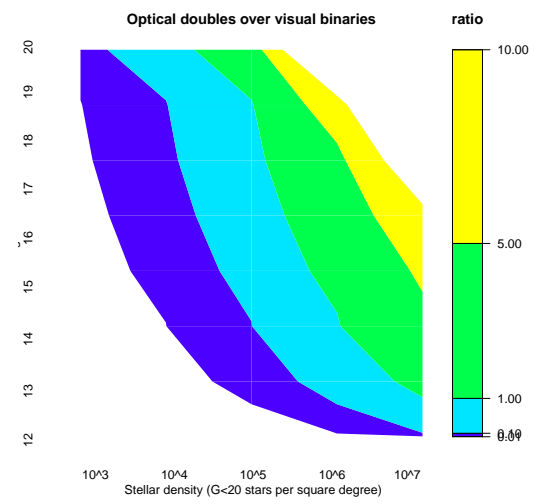

FIGURE 8. Ratio of the number of detected optical doubles over visual binaries, as a function of the primary magnitude and stellar density at magnitude 20 .

Finally, only the "static" part of the multiple star simulations has been described here. Beyond the Galactic properties of multiple stars, the astrometric (position, proper motion), spectroscopic (radial velocity) and photometric (eclipses) effects of these objects on the transit observations have to be taken into account: in the course of the simulations of Gaia observations, the orbits are thus computed, the positions/velocities/photometry of both components are modified accordingly.

Both the static and dynamic part of the multiple stars simulation thus allow to estimate as precisely as possible the output of the mission with the current knowledge of the 
binary properties. When the actual output data is produced, it can also be used to infer the actual properties of the stellar multiplicity.

\section{REFERENCES}

1. F. Mignard, et al., "Gaia: organisation and challenges for the data processing," vol. 248 of $I A U$ Symposium, pp. 402-403.

2. D. Pourbaix, "Screening and modelling the double and multiple stars observed by Gaia", This conference

3. F. Arenou \& S. Söderhjelm, "Gaia Data Reduction Tasks for Double and Multiple Stars", in "The Three-Dimensional Universe with Gaia” edited by C. Turon, K.S. O'Flaherty, M.A.C. Perryman, 2005, ESA SP-576, pp.547-550

4. Robin, A.C., Reylé, C., Derrière, S., Picaud, S., 2003, A\&A, 409, 523, ADS Link

5. F. Arenou, Java simulation of binaries, Tech. Rep. DMS-FA-03 (GAIA-C4-TN-OPM-FA-008-3), Observatoire de Paris / CNRS (2003), URL http://wwwhip.obspm.fr/gaia/dms/texts/ DMS-FA-03.pdf.

6. A. Duquennoy, and M. Mayor, A\&A 248, 485-524 (1991).

7. S. Söderhjelm, Theoretical modelling of 'observational' double-star distribution functions., Tech. Rep. DMS-SS-005, Lund Observatory (2004), URL http: //wwwhip.obspm.fr/gaia/dms/ texts/DMS05b.pdf

8. M. B. N. Kouwenhoven, A. G. A. Brown, S. P. Goodwin, S. F. Portegies Zwart, and L. Kaper, Astronomische Nachrichten 329, 984-987 (2008), 0811.3092.

9. J. Voyez, Simulation d'étoiles binaires pour la préparation de la mission spatiale gaia, Tech. rep., Stage de Licence 3 Parcours Magistère, Université Paris Diderot (2008).

10. C. J. Lada, ApJ 640, L63-L66 (2006), arXiv: astro-ph/ 0601375

11. A. Tokovinin, "Multiple Stars: Designation, Catalogues, Statistics," in Multiple Stars Across the H-R Diagram, ESO Astrophysics Symposia, 2008, pp. 38-+.

12. A. Eggenberger, J.-L. Halbwachs, S. Udry, and M. Mayor, "Statistical properties of an unbiased sample of F7-K binaries: towards the long-period systems," in Revista Mexicana de Astronomia y Astrofisica Conference Series, edited by C. Allen, and C. Scarfe, 2004, vol. 21 of Revista Mexicana de Astronomia y Astrofisica Conference Series, pp. 28-32.

13. X. Delfosse, J. Beuzit, L. Marchal, X. Bonfils, C. Perrier, D. Ségransan, S. Udry, M. Mayor, and T. Forveille, "M dwarfs binaries: Results from accurate radial velocities and high angular resolution observations," in Spectroscopically and Spatially Resolving the Components of the Close Binary Stars, edited by R. W. Hilditch, H. Hensberge, \& K. Pavlovski, 2004, vol. 318 of Astronomical Society of the Pacific Conference Series, pp. 166-174.

14. L. M. Close, N. Siegler, M. Freed, and B. Biller, ApJ 587, 407-422 (2003), arXiv: astro-ph/ 0301095 .

15. I. N. Reid, K. L. Cruz, A. J. Burgasser, and M. C. Liu, AJ 135, 580-587 (2008).

16. A. J. Burgasser, J. D. Kirkpatrick, I. N. Reid, M. E. Brown, C. L. Miskey, and J. E. Gizis, ApJ 586, 512-526 (2003), arXiv:astro-ph/0211470

17. M. F. Sterzik, and R. H. Durisen, "Are Binary Separations related to their System Mass?," in Revista Mexicana de Astronomia y Astrofisica Conference Series, edited by C. Allen, and C. Scarfe, 2004, vol. 21 of Revista Mexicana de Astronomia y Astrofisica Conference Series, pp. 58-62.

18. N. Shatsky, and A. Tokovinin, Astronomy and Astrophysics 382, 92-103 (2002), arXiv: astro-ph/0109456.

19. M. B. N. Kouwenhoven, A. G. A. Brown, H. Zinnecker, L. Kaper, and S. F. Portegies Zwart, Astronomy and Astrophysics 430, 137-154 (2005), arXiv: astro-ph/0410106

20. M. B. N. Kouwenhoven, A. G. A. Brown, S. F. Portegies Zwart, and L. Kaper, Astronomy and Astrophysics 474, 77-104 (2007), arXiv: 0707.2746

21. H. A. Kobulnicky, and C. L. Fryer, Astrophysical Journal 670, 747-765 (2007).

22. B. D. Mason, D. R. Gies, W. I. Hartkopf, W. G. Bagnuolo, Jr., T. ten Brummelaar, and H. A. McAlister, Astronomical Journal 115, 821-+ (1998). 
23. J. L. Halbwachs, M. Mayor, S. Udry, and F. Arenou, Astronomy and Astrophysics 397, 159-175 (2003).

24. I. Thies, and P. Kroupa, ApJ 671, 767-780 (2007), 0708.1764

25. P. P. Eggleton, and A. A. Tokovinin, MNRAS 389, 869-879 (2008), arXiv: 0806.2878 ,

26. H. A. Abt, A. E. Gomez, and S. G. Levy, Astrophysical Journal Supplement 74, 551-573 (1990).

27. B. Famaey, A. Jorissen, X. Luri, M. Mayor, S. Udry, H. Dejonghe, and C. Turon, A\&A 430, 165-186 (2005), arXiv:astro-ph/0409579

28. J. Mermilliod, J. Andersen, D. W. Latham, and M. Mayor, A\&A 473, 829-845 (2007).

29. A. Frankowski, B. Famaey, S. van Eck, M. Mayor, S. Udry, and A. Jorissen, A\&A 498, 479-488 (2009), 0901.0937

30. T. A. Fleming, S. L. Snowden, E. Pfeffermann, U. Briel, and J. Greiner, A\&A 316, 147-154 (1996).

31. M. A. Barstow, J. B. Holberg, T. A. Fleming, M. C. Marsh, D. Koester, and D. Wonnacott, MNRAS 270, 499-+ (1994).

32. J. B. Holberg, E. M. Sion, T. Oswalt, G. P. McCook, S. Foran, and J. P. Subasavage, AJ 135, 1225$1238(2008)$.

33. M. L. Pretorius, C. Knigge, D. O'Donoghue, J. P. Henry, I. M. Gioia, and C. R. Mullis, MNRAS 382, 1279-1288 (2007), 0709.1887.

34. S. Catalán, J. Isern, E. García-Berro, and I. Ribas, Journal of Physics Conference Series 172, 012007 $+(2009)$.

35. A. Tokovinin, S. Thomas, M. Sterzik, and S. Udry, "Tertiary Companions to Close Spectroscopic Binaries," in Multiple Stars Across the H-R Diagram, edited by S. Hubrig, M. Petr-Gotzens, \& A. Tokovinin, 2008, pp. 129-+.

36. A. J. Burgasser, I. N. Reid, N. Siegler, L. Close, P. Allen, P. Lowrance, and J. Gizis, Protostars and Planets V pp. 427-441 (2007), arXiv:astro-ph/0602122

37. S. Söderhjelm, A\&A 463, 683-691 (2007).

38. A. Tokovinin, MNRAS 389, 925-938 (2008), 0806.3263 .

39. H. Bouy, E. Martín, W. Brandner, and J. Bouvier, Astronomische Nachrichten 326, 969-973 (2005).

40. A. Tokovinin, "Statistics of multiple stars," in Revista Mexicana de Astronomia y Astrofisica Conference Series, edited by C. Allen \& C. Scarfe, 2004, vol. 21 of Revista Mexicana de Astronomia y Astrofisica, vol. 27, pp. 7-14.

41. H. A. Abt, and C. J. Corbally, ApJ 541, 841-848 (2000).

42. C. Allen, M. Tapia, and L. Parrao, Revista Mexicana de Astronomia y Astrofisica, vol. 3 3, 119-+ (1977).

43. H. A. Abt, ApJ 629, 507-511 (2005).

44. H. A. Abt, ApJ 651, 1151-1154 (2006).

45. B. Tingley, G. Sadowski, and C. Siopis, "Towards a fully automated eclipsing binary solver for Gaia," in IAU Symposium, 2009, vol. 253 of IAU Symposium, pp. 402-403.

46. P. North, Ecole de Goutelas \#23, CNRS, 22-26 May 2000, edited by D. Egret, J.-L. Halbwachs, and J.-M. Hameury. Publisher: Société Francaise d'Astronomie et d'Astrophysique (SF2A) 23, 67-+ (2001).

47. S. R. Kane, and K. von Braun, ApJ 689, 492-498 (2008), 0808 .1890.

48. F. Arenou, Recent simulations for the compression and on-board detection studies, Tech. Rep. PDH2-3120-TN-001.2 (GAIA-CA-TN-OPM-FA-046-2), Observatoire de Paris / CNRS (2007), URL http://www.rssd.esa.int/llink/livelink/open/2733272.

49. Henry, T.J., Jao, W., Subasavage, J.P., et al., 2006, AJ 132, 2360, ADS Link (2006).

50. S. Söderhjelm, Simulation results for gaia double stars - IV, Tech. Rep. DMS-SS-04, Lund Observatory (2003), URL http://wwwhip.obspm.fr/gaia/dms/texts/DMS 04 .ps. 